Virginia Commonwealth University vCU Scholars Compass

2012

\title{
Optimizing fiber cross-sectional shape for improving stability of air-water interface over superhydrophobic fibrous coatings
}

B. Emami

Virginia Commonwealth University

Hooman Vahedi Tafreshi

Virginia Commonwealth University, htafreshi@vcu.edu

Follow this and additional works at: http://scholarscompass.vcu.edu/egmn_pubs

Part of the Mechanical Engineering Commons, and the Nuclear Engineering Commons

Emami, B. and Tafreshi, H.V. Optimizing fiber cross-sectional shape for improving stability of air-water interface over superhydrophobic fibrous coatings. Applied Physics Letters, 100, 193104 (2012). Copyright (C 2012 AIP Publishing LLC.

\section{Downloaded from}

http://scholarscompass.vcu.edu/egmn_pubs/15

This Article is brought to you for free and open access by the Dept. of Mechanical and Nuclear Engineering at VCU Scholars Compass. It has been accepted for inclusion in Mechanical and Nuclear Engineering Publications by an authorized administrator of VCU Scholars Compass. For more information, please contact libcompass@vcu.edu. 


\title{
Optimizing fiber cross-sectional shape for improving stability of air-water interface over superhydrophobic fibrous coatings
}

\author{
B. Emami and H. Vahedi Tafreshi ${ }^{\mathrm{a})}$ \\ Department of Mechanical and Nuclear Engineering, Virginia Commonwealth University, Richmond, \\ Virginia 23284-3015, USA
}

(Received 11 February 2012; accepted 19 April 2012; published online 7 May 2012)

\begin{abstract}
In this letter, a mathematical force-balance formulation is developed that can be used to predict the critical pressure, the hydrostatic pressure above which the surface starts to depart from the non-wetting state, for superhydrophobic surfaces comprised of highly aligned fibers (e.g., biased AC-electrospun coatings) with arbitrary cross-sectional shapes. We have also developed a methodology for optimizing the fiber cross-sections to maximize the critical pressure of the surface, using the Euler-Lagrange equation. A case study is presented to better demonstrate the application of our method. (C) 2012 American Institute of Physics. [http://dx.doi.org/10.1063/1.4711800]
\end{abstract}

Superhydrophobicity is brought about by combining micro- and nano-scale surface roughness with the hydrophobicity of the bulk material, resulting in static contact angles greater than $150^{\circ} .{ }^{1,2}$ This is due to the formation of entrapped air pockets in the micro- and/or nano-pores of the surface, leading to a reduced solid surface area in contact with water-an effect that can lead to reduced skin-friction drag on submerged moving objects. ${ }^{3}$ Superhydrophobic surfaces, however, are reported to transition from the Cassie (non-wetting) ${ }^{4}$ state to the Wenzel (fully wetted) ${ }^{5}$ state if exposed to excessive hydrostatic pressures. ${ }^{6-9}$ There have been recent studies dedicated to developing mathematical formulations to predict the so-called "critical pressure," the pressure above which a superhydrophobic surface starts departing from the Cassie state. ${ }^{10-15}$

Producing superhydrophobic surfaces made up of electrospun fibers has been reported in variety of studies conducted in the past decade (see Ref. 16 for a review). In a conventional electrospinning process, the fibers are ejected from a nozzle and are drawn into thinner strands by aerodynamic and electrostatic forces as they travel toward the collector. The fibers eventually deposit onto a substrate attaining random in-plane orientations (see Ref. 17 for a review). The first attempt to predict critical pressure for electrospun surfaces was made by Tuteja et al. ${ }^{18}$ These authors considered a simplified geometry of equally spaced parallel fibers with circular cross-sections placed on a flat surface, and developed two criteria for the evaluating the stability of the air-water interface in the grooves formed by the fibers under elevated pressures. Although it was not discussed by Tuteja et al., ${ }^{18}$ the formulations given by these authors were actually more appropriate for fibrous surfaces comprised of aligned fibers, as opposed to those having random in-plane orientations. Such anisotropic fibrous structures can be produced by modifying the traditional electrospinning process. Among different techniques, the biased AC-electrospinning of Tepper and his co-workers ${ }^{19,20}$ has shown promising con-

\footnotetext{
a) Author to whom correspondence should be addressed. Electronic mail: htafreshi@vcu.edu. Tel.: 804-828-9936. Fax: 804-827-7030. URL: http://www.people.vcu.edu/ htafreshi/.
}

trol over fiber orientation, as can be seen in Figure 1. In a recent study, we simulated the influence of the fibers' inplane orientation on the critical pressure and discussed the role of microstructural parameters of a fibrous surface on its resistance against elevated hydrostatic pressures using the so-called full morphology method. ${ }^{21}$ The focus of the current paper, on the other hand, is on the effects of fibers' crosssectional shape on the critical pressure. In particular, we have developed a rigorous mathematical method to predict the critical pressure of electrospun superhydrophobic coatings. As the 3-D morphology of fibrous coatings with random fiber orientation is prohibitively complicated from a mathematical point of view, our formulations are developed in two dimensions and so are more accurate for coatings made up of aligned fibers, e.g., biased AC-spun fibrous coatings. The mathematical framework developed in this study, however, allows the fibers to have any arbitrary crosssection, and more importantly, is capable of optimizing the fibers' cross-sectional shape to maximize the critical pressure of the coatings. Such information is particularly important for designing fibrous superhydrophobic coatings for underwater applications where resistance against elevated hydrostatic pressures is crucially important. ${ }^{21-27}$

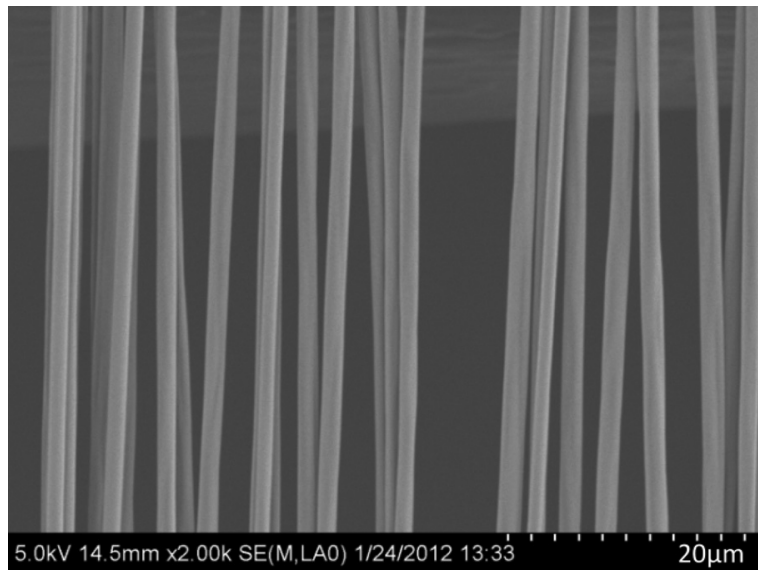

FIG. 1. An example of a fibrous coating consisting of highly aligned polystyrene fibers produced via biased AC-electrospinning. 
Let us consider a groove formed between two identical fibers with arbitrary cross-sectional shapes as shown in Figure 2. Consider the groove to have a width of $2 w$, a depth of $h$, and an arbitrary wall shape $y=g(x)$. We assume that the fiber surface is smooth, and so the air-water interface forms an equilibrium contact angle $\theta$ with the groove walls. At any given hydrostatic pressure, the interface intersects with the walls at a point $q$, corresponding to a coordinate $\left(x_{q}, g\left(x_{q}\right)\right)$, as shown in Figure 2. Applying balance of forces across the air-water interface in the $y$-direction, we obtain

$$
P\left(w-g\left(x_{q}\right)\right)=-\sigma \sin (\alpha+\theta),
$$

where $\sigma$ is the air-water surface tension, $\alpha$ is the local angle between the wall and the $y$-axis, and so the derivative of $g$ with respect to $x, g^{\prime}$, equals $\cot \alpha$. Let $\vec{n}$ be the unit vector normal to the groove wall. It can be observed from Figure 2 that $\vec{n} \cdot \vec{i}=-\cos \alpha$ and $\vec{n} \cdot \vec{j}=\sin \alpha$, where $\vec{i}$ and $\vec{j}$ are the unit vectors in $x$ and $y$ directions, respectively. On the other hand, it can be shown that the elements of the unit vector normal to a smooth curve $y=g(x)$ in $x$ and $y$ directions are, respectively, $-g^{\prime}\left(1+g^{\prime 2}\right)^{-1 / 2}$ and $\left(1+g^{\prime 2}\right)^{-1 / 2}$. Thus, $\cos \alpha$ $=g^{\prime}\left(1+g^{\prime 2}\right)^{-1 / 2}$ and $\sin \alpha=\left(1+g^{\prime 2}\right)^{-1 / 2}$. Equation (1) then becomes

$$
P=\sigma\left(g\left(x_{q}\right)-w\right)^{-1}\left[1+g^{\prime 2}\left(x_{q}\right)\right]^{-1 / 2}\left(g^{\prime}\left(x_{q}\right) \sin \theta+\cos \theta\right) .
$$

Note that in the above equation, $P$ and $g$ correspond to the point $q$ with coordinates $\left(x_{q}, g\left(x_{q}\right)\right)$, where the interface intersects with the wall. As the hydrostatic pressure increases, the interface moves downward and hence $x_{q}$ increases. Equation (2) yields the hydrostatic pressure corresponding to a given intersection point $q$. The critical pressure is the maximum hydrostatic pressure that the groove can sustain, and it occurs at a point $\left(x_{q, c r}, g\left(x_{q, c r}\right)\right)$ where the derivative of $P$ with respect to $x_{q}$ goes to zero (i.e., $\left.\frac{d P}{d x_{q}}\right|_{x_{q, c r}}=0$ ). This yields

$$
\begin{aligned}
& g^{\prime}\left(x_{q}\right)\left[1+g^{\prime 2}\left(x_{q}\right)\right]\left[\sin \theta g^{\prime}\left(x_{q}\right)+\cos \theta\right] \\
& +\left(g\left(x_{q}\right)-w\right) g^{\prime \prime}\left(x_{q}\right)\left[\cos \theta g^{\prime}\left(x_{q}\right)-\sin \theta\right]=0 .
\end{aligned}
$$

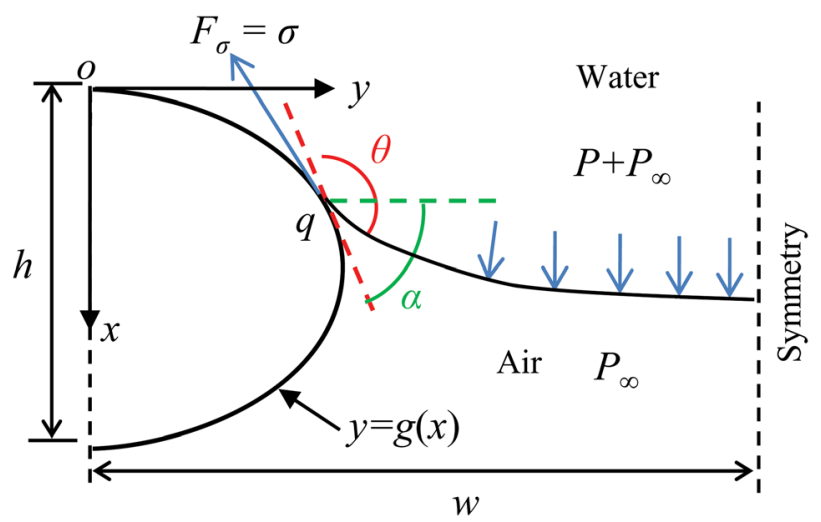

FIG. 2. Schematic of a groove with an arbitrary wall shape $y=g(x)$. Only half of the groove is shown due to symmetry.
The solution of the above nonlinear equation is the $x$-coordinate $x_{q, c r}$ of the interface-wall intersection point that corresponds to the critical pressure. The critical pressure $P_{c r}$ can then be calculated by inserting $x_{q, c r}$ into Eq. (2), $P_{c r}=P\left(x_{q, c r}\right)$. Here, we assume that the air-water interface does not reach the groove bottom before the critical pressure is reached. This assumption is justified by considering the fact that surfaces made of parallel fibers (as opposed to those comprised of randomly oriented fibers, such as DCelectrospun surfaces) tend to have relatively high solid volume fractions (i.e., fibers tend to pack closely near one another as can be seen in Figure 1). For fibrous surfaces with high solid volume fraction, it is less probable for the interface to reach the bottom of the groove before a critical pressure is reached. This has been fully discussed in our previous publication by Bucher et al. ${ }^{21}$ (see Figure 5(a) in Ref. 21 where the robustness height method of Ref. 18 is compared to our force balance method).

To better demonstrate the application of our force balance method in critical pressure prediction, we have considered the case of a superhydrophobic surface comprised of aligned fibers with circular cross sections. When the walls of the groove are circular, then $g(x)=\sqrt{\frac{h^{2}}{4}-\left(x-\frac{h}{2}\right)^{2}}$. Here, we assume that $w=h=1 \mu \mathrm{m}$, and that $\theta=110^{\circ}$. Figure 3 shows the hydrostatic pressure $P$ versus the $x$-coordinate of the interface-wall intersection point calculated from Eq. (2). Our formulations for this surface result in a critical pressure
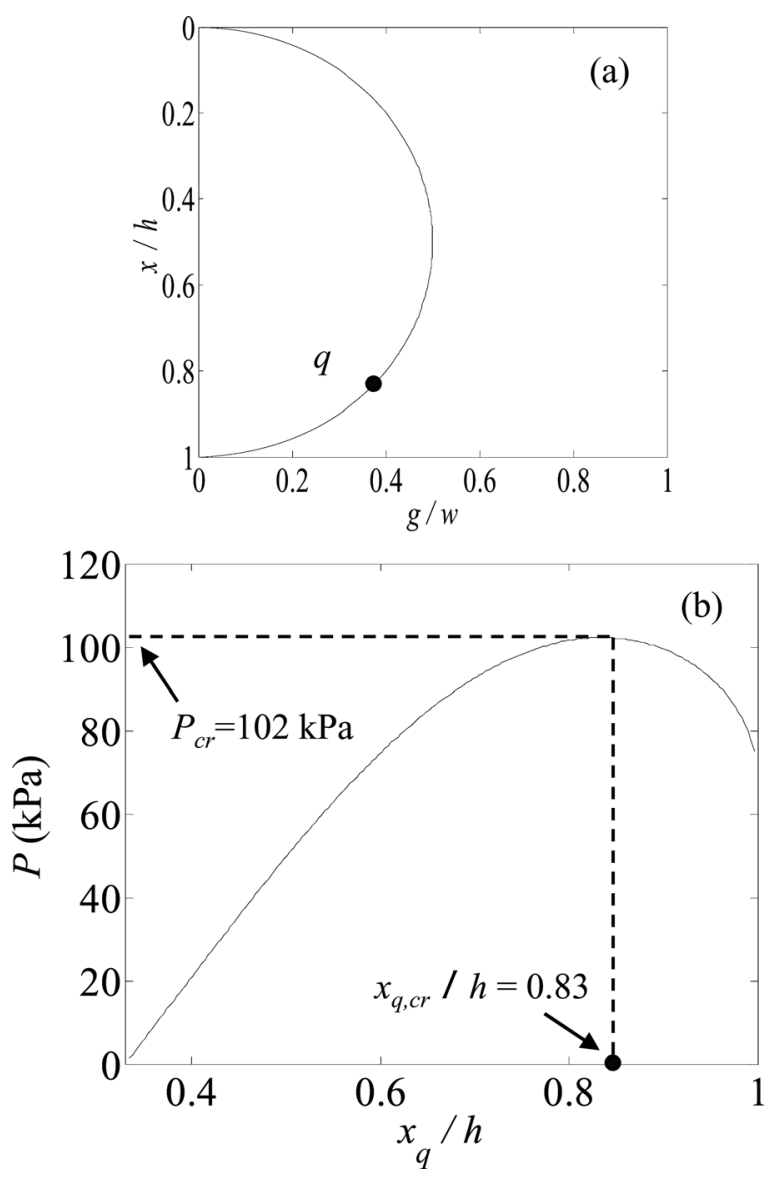

FIG. 3. Hydrostatic pressure versus the corresponding $x_{q}$ normalized by the groove depth $h$ for a groove with semi-spherical walls; $w=h=1 \mu \mathrm{m}$. 
$P_{c r}$ of $102 \mathrm{kPa}$, and predict the intersection point to stabilize at $x_{q, c r}=0.83 h$, as calculated from Eq. (3). As mentioned earlier, the 2-D analysis of aligned fibers with circular cross sections was originally studied in Ref. 18. The authors in Ref. 18 defined two criteria namely "robustness height" $P_{H}$ and "robustness angle" $P_{\theta}$ formulas, for determining the critical pressure of a superhydrophobic surface, and recommended the minimum value between the two be taken as the critical pressure the surface. For the dimensions considered here, $P_{H}$ and $P_{\theta}$ are found to be 390 and $136 \mathrm{kPa}$, respectively, indicating a critical pressure of $136 \mathrm{kPa}$ for the surface (see Ref. 18 for the equations), in good agreement with the predictions obtained from Eq. (2). In fact, the special case of superhydrophobic surfaces with aligned fibers having circular cross sections has been fully discussed in our previous work by Bucher et al., ${ }^{21}$ and a series of comparisons have been made between our force balance equations and those given in Ref. 18.

In this paper, we also proposed a method for optimizing the fiber cross-sectional shape to maximize the critical pressure for a given groove of dimensions (width $w$ and depth $h$ ) and material (contact angle $\theta$ ). As was shown earlier, critical pressure depends on the $y$-component of the capillary force $F_{\sigma}$, and on the $y$-component of the force exerted by hydrostatic pressure. It can be shown that the $x$-component of the capillary force vanishes at any point on the groove wall where $g^{\prime}=\tan \theta$. Because the value of the capillary force is constant, $F_{\sigma}=\sigma$, this means that the $y$-component of the capillary force is maximal where $g^{\prime}=\tan \theta$. On the other hand, the $y$-component of the hydrostatic force exerted on the interface is minimal when the area $w-g$ is minimal (see Figure 2). Therefore, an optimal grove shape which yields the maximum possible critical pressure should correspond to a minimum $\left|g^{\prime}-\tan \theta\right|$ and $|w-g|$. Such an optimized groove profile $g(x)$ is then the minimal path of the functional $I[g]$, defined as

$$
I[g] \equiv \int_{0}^{h} L\left(g, g^{\prime}\right) d x,
$$

where $L\left(g, g^{\prime}\right) \equiv(g(x)-w)^{2}\left(g^{\prime}(x)-\tan \theta\right)^{2}$. This is because the minimal of the above integral corresponds to a minimum $\left|g^{\prime}-\tan \theta\right|$ and $|w-g|$. Here, we limit the groove wall shapes to smooth curves with the fixed endpoints $g(x=0)=g(x=h)=0$ (see Figure 2). To find the path $g^{*}(x)$ at which the above functional is minimum, we used variational analysis. ${ }^{28}$ If the groove shape $g$ changes infinitesimally to $g+\delta g$, the functional $I$ changes to $I+\delta I$. Substituting $g+\delta g$ into Eq. (4), using Taylor series and neglecting the second or higher order terms, one obtains

$$
\delta I=\int_{0}^{h}\left(\frac{\partial L}{\partial g} \delta g+\frac{\partial L}{\partial g^{\prime}} \delta g^{\prime}\right) d x
$$

and using integration by parts,

$$
\delta I=\left(\frac{\partial L}{\partial g^{\prime}} \delta g\right)_{x=0}^{x=h}+\int_{0}^{h}\left[\frac{\partial L}{\partial g}-\frac{d}{d x}\left(\frac{\partial L}{\partial g^{\prime}}\right)\right] \delta g d x .
$$

At the optimal wall shape, $g=g^{*}$, the variation in the functional $\delta I$ vanishes. Because $g$ is fixed at both $x=0$ and $x=h$, the first term on the right hand side of Eq. (6) also vanishes. Therefore,

$$
\frac{\partial L}{\partial g^{*}}=\frac{d}{d x}\left(\frac{\partial L}{\partial g^{*^{\prime}}}\right)
$$

This is in fact the Euler-Lagrange equation, ${ }^{25}$ the solution of which is the minimal path $g^{*}(x)$. Substituting $L\left(g^{*}, g^{*^{\prime}}\right)$ $=\left(g^{*}(x)-w\right)^{2}\left(g^{*^{\prime}}(x)-\tan \theta\right)^{2}$ into Eq. (7), one obtains

$$
\left(g^{*}-w\right) g^{* \prime \prime}+g^{*^{\prime 2}}-\tan ^{2} \theta=0,
$$

which is subject to the boundary conditions $g^{*}(x=0)=g^{*}$ $(x=h)=0$. Because the above differential equation does not have a unique solution, we further impose a constraint

$$
\int_{0}^{h} g^{*}(x) d x=A_{0}
$$

which means that the area below the curve $y=g(x)$ is fixed to a constant value $A_{0}$. This means that the cross-sectional area of the fibers is held constant during the fiber profile optimization process. In other words, our optimization methodology is developed for coatings with identical mass of
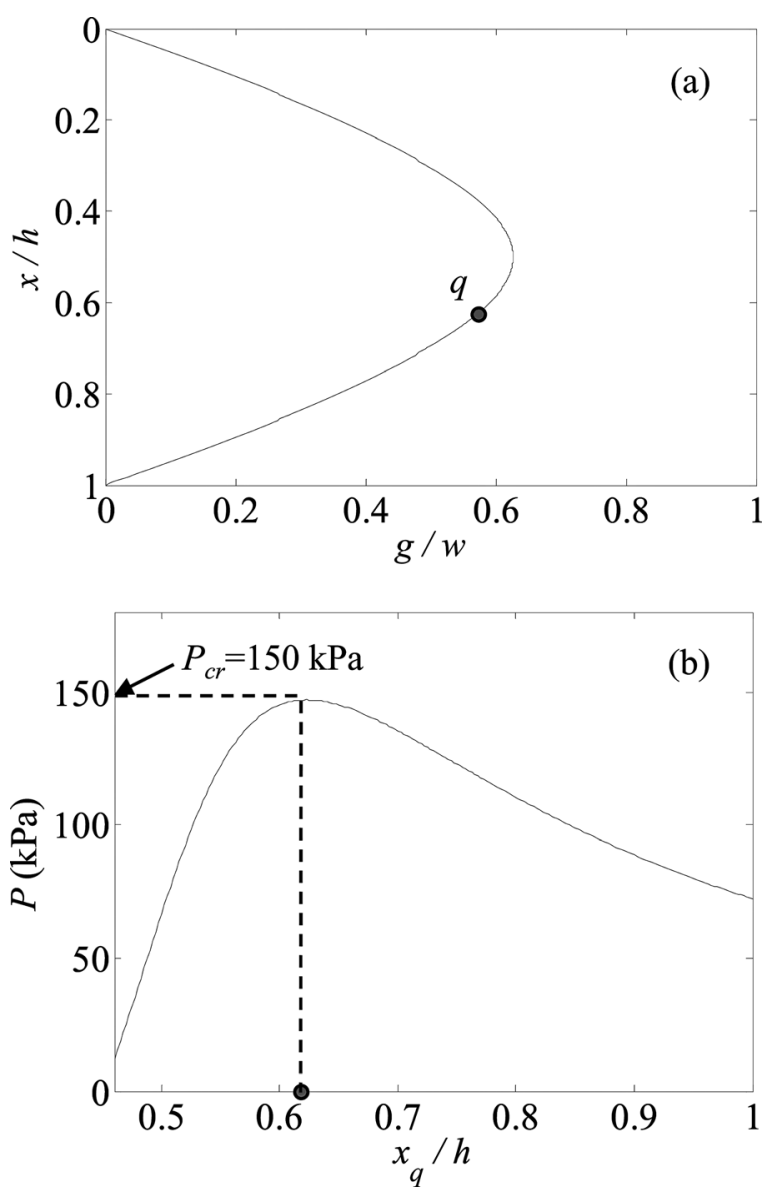

FIG. 4. (a) Optimized wall shape profile for $w=h=1 \mu \mathrm{m}$. (b) Hydrostatic pressure versus the corresponding $x_{q}$ normalized by the groove depth $h$ for the groove with optimized wall shapes. 
deposited fibers (i.e., optimizing the cross-sectional shape of the fibers while keeping the coatings' porosity constant).

For demonstration purposes, we have calculated the optimal cross-section of superhydrophobic aligned fibers with specifications similar to those of the circular fibers discussed earlier, namely, $w=h=1 \mu \mathrm{m}, \quad \theta=110^{\circ}$, and $A_{0}=\pi h^{2} / 8$ (half the cross-sectional area of a circular fiber). We used the FlexPDE finite element code from PDESolutions, Inc., to solve Eq. (6) along with the above boundary conditions and the integral constraint. The numerical solution was run on a workstation with a dual-core $2.4 \mathrm{GHz} \mathrm{CPU}$ and 4 GB of RAM; the numerical solution took less than a minute to converge. Careful attention was paid to ensure that the results of our calculation are not dependent on the choice of the mesh size. Figure 4(a) shows the calculated profile of the optimal fiber cross-section. Equation (2) is used again to calculate the hydrostatic pressure $P$ versus the $x$-coordinate of the interface-wall intersection point $x_{q}$ (see Figure 4(b)). As can be seen, the superhydrophobic surface comprised of aligned fibers with optimized cross-sectional profile has a critical pressure of $150 \mathrm{kPa}$, which is much higher than that of the surface made up of circular fibers.

In summary, a rigorous mathematical framework is developed in this work to predict the critical pressure of a superhydrophobic surface comprised of aligned fibers with arbitrary cross-sections. In addition, we have established an optimization methodology that can potentially be used to design superhydrophobic surfaces with better resistance against elevated hydrostatic pressures.

Financial support for this work was provided by VCU Presidential Research Incentive Program (PRIP). The authors would like to acknowledge Professor Gary Tepper of VCU School of Engineering for Fig. 1.
${ }^{1}$ J. Wang, F. Liu, H. Chen, and D. Chen, Appl. Phys. Lett. 95, 084104-1 (2009).

${ }^{2}$ J. P. Rothstein, Annu. Rev. Fluid Mech. 42, 89 (2010).

${ }^{3}$ A. Marmur, Langmuir 22, 1400-1402 (2006).

${ }^{4}$ A. B. D. Cassie and S. Baxter, Trans. Faraday Soc. 40, 546 (1944).

${ }^{5}$ R. N. Wenzel, Ind. Eng. Chem. 28, 988 (1936).

${ }^{6}$ B. Liu and F. F. Lange, J. Colloid Interface Sci. 298, 899 (2006).

${ }^{7}$ Q. S. Zheng, Y. Yu, and Z. H. Zhao, Langmuir 21, 12207 (2005).

${ }^{8}$ X. Sheng and J. Zhang, Colloids Surf., A 377, 374 (2011).

${ }^{9}$ A. Lafuma and D. Quere, Nature Mater. 2, 457 (2003).

${ }^{10}$ C. W. Extrand, Langmuir 20, 5013 (2004).

${ }^{11}$ C. W. Extrand, Langmuir 27, 6920 (2011).

${ }^{12}$ B. Emami, T. Bucher, H. V. Tafreshi, M. Gad-el-Hak, and G. C. Tepper, Colloids Surf., A 385, 95 (2011).

${ }^{13}$ B. Emami, H. V. Tafreshi, M. Gad-el-Hak, and G. C. Tepper, Appl. Phys. Lett. 98, 203106 (2011).

${ }^{14}$ B. Emami, H. V. Tafreshi, M. Gad-el-Hak, and G. C. Tepper, Appl. Phys. Lett. 100, 013104 (2012).

${ }^{15}$ B. Emami, H. V. Tafreshi, M. Gad-el-Hak, and G. C. Tepper, J. Appl. Phys. 111, 064325 (2012).

${ }^{16}$ X. F. Wang, B. Ding, J. Y. Yu, and M. R. Wang, Nanotoday 6, 510 (2011).

${ }^{17}$ D. H. Reneker and A. L. Yarin, Polymer 49, 2387 (2008).

${ }^{18}$ A. Tuteja, W. Choi, J. M. Mabry, G. H. McKinley, and R. E. Cohen, Proc. Natl. Acad. Sci. U.S.A. 105, 18200 (2008).

${ }^{19}$ R. Kessick, J. Fenn, and G. C. Tepper, Polymer 45, 2981 (2004).

${ }^{20}$ S. Sarkar, S. C. Deevi, and G. Tepper, Macromol. Rapid Commun. 28, 1034 (2007).

${ }^{21}$ T. M. Bucher, B. Emami, H. V. Tafreshi, M. Gad-el-Hak, and G. C. Tepper, Phys. Fluids 24, 022109 (2012).

${ }^{22}$ M. S. Bobji, S. V. Kumar, A. Asthana, and R. N. Govardhan, Langmuir 25, 12120 (2009).

${ }^{23}$ R. Poetes, K. Holtzmann, K. Franze, and U. Steiner, Phys. Rev. Lett. 105, 166104 (2010).

${ }^{24}$ G. Mchale, M. I. Newton, and N. J. Shirtcliff, Soft Matter 6, 714 (2010).

${ }^{25}$ P. Forsberg, F. Nikolajeff, and M. Karlsson, Soft Matter 7, 104 (2011).

${ }^{26}$ M. A. Samaha, F. O. Ochanda, H. V. Tafreshi, G. C. Tepper, and M. Gadel-Hak, Rev. Sci. Instrum. 82, 0451091 (2011).

${ }^{27}$ M. A. Samaha, H. V. Tafreshi, and M. Gad-el-Hak, Colloids Surf., A 399, 62-70 (2012).

${ }^{28}$ O. Buhler, A Brief Introduction to Classical, Statistical, and Quantum Mechanics (American Mathematical Society, 2000). 